\title{
FLOSS Survey 2013? A Proposal for Comprehensive Survey on FLOSS Developers
}

\author{
Masayuki Hatta \\ Surugadai University, Faculty of Economics, Room 710E, \\ $10^{\text {th }}$ Floor, The Di-ni Bldg, 698 Asu, Hannou Saitama, Japan 357-8555 \\ hatta.masayuki@surugadai.ac.jp \\ http: //about.me/mhatta
}

The year 2013 will mark the 10th anniversary of the renowned FLOSS surveys[1]. They were the first international efforts to conduct online (and offline) questionnaire surveys to collect comprehensive data on FLOSS developers, such as who they were, what motivated them, how they were organized, then aim at picturing an overview of what the FLOSS (Free/Libre/Open Source Software) development was all about. The outcome of FLOSS surveys has been considered as one of the most reliable sources on FLOSS ecosystem for researchers (especially in the realm of social sciences) who are interested in the FLOSS development process at large.

The FLOSS surveys provide us quite a complete outlook of the FLOSS world at that time. However, the following ten years since then were possibly the most rapidly evolving, dramatically changing days of ICT industries -- who could expect in those days, an yet another small tech startup which bore the strange name Google or an ailing niche computer maker named Apple would become the pinnacle of the world? It is highly possible that the landscape of FLOSS development is also radically different from the good old days of 10 years ago. Ever since, many researchers conducted smaller surveys, but most of them tended to be quite specific to their own research interest and not comprehensive, thus cannot be compared with the result of FLOSS 2002/2003 directly in its entirety.

In my poster, I propose a plan to conduct the second FLOSS survey. It will update the FLOSS 2002/2003 and give us a renewed view of what has become of the FLOSS world. Also, I intend to establish a good practice to conduct an online survey, using purely Free / Open Source Software.

\section{Reference}

1. http://flossproject.org/report/ 